\title{
Navier-Stokes Equations with Degenerate Viscosity, Vacuum and Gravitational Force
}

\author{
RenJun DuAn \\ Department of Mathematics, City University of Hong Kong \\ 83 Tat Chee Avenue, Kowloon, Hong Kong, P.R. China \\ Email: renjun_duan@yahoo.com.cn
}

TONG YANG

Liu Bie Ju Centre of Mathematics, City University of Hong Kong

83 Tat Chee Avenue, Kowloon, Hong Kong, P.R. China

Email: matyang@cityu.edu.hk

ChangJiang Zhu

Laboratory of Nonlinear Analysis, Department of Mathematics Central China Normal University, Wuhan 430079, P.R. China

Email: cjzhu@mail.ccnu.edu.cn

\begin{abstract}
The global existence of weak solutions to the compressible Navier-Stokes equations with vacuum attracts many research interests recently. For the isentropic gas, the viscosity coefficient depends on density function from physical point of view. When the density function connects to vacuum continuously, the vacuum degeneracy gives some analytic difficulties in proving global existence. In this paper, we consider this case with gravitational force and fixed boundary condition. By giving a series of $a$ priori estimates on the solution coping with the degeneracy of vacuum, gravitational force and boundary effect, we give global existence and uniqueness results similar to the case without force and boundary.
\end{abstract}

Key words: Navier-Stokes equations, vacuum, a priori estimates, global existence.

\section{Introduction}

Consider the one-dimensional compressible Navier-Stokes equations for isentropic flow with gravitational force in Eulerian coordinates:

$$
\left\{\begin{array}{l}
\rho_{\tau}+(\rho u)_{\xi}=0, \\
(\rho u)_{\tau}+\left(\rho u^{2}+P(\rho)\right)_{\xi}=\left(\mu u_{\xi}\right)_{\xi}+\rho g, \quad \text { in } y(\tau)<\xi<0, \quad \tau>0,
\end{array}\right.
$$


with initial data

$$
\rho(\xi, 0)=\rho_{0}(\xi), \quad u(\xi, 0)=u_{0}(\xi), \quad \text { on } \quad y(0) \leq \xi \leq 0,
$$

and boundary condition

$$
u(1, \tau)=0 .
$$

Here the unknown functions $\rho=\rho(\xi, \tau)$ and $u=u(\xi, \tau)$ represent the density and velocity respectively. $P(\rho)=A \rho^{\gamma}$ is the pressure with $A$ being a positive constant and $\gamma>1$, $\mu \geq 0$ is the viscosity coefficient, and $g>0$ is the gravitational constant. $y(\tau)$ is the vacuum boundary, i.e., the particle path separating the gas and the vacuum, satisfying

$$
\frac{d y}{d \tau}=u(y(\tau), \tau), \quad \rho(y(\tau), \tau)=0
$$

Our main concern here is the global existence and the uniqueness of weak solutions with some regularity for the description on the vacuum boundary to the above initial boundary value problem when the viscosity coefficient depends on the density function. It is a continuation of works in $[26,28,29,30]$ which are about the case when no boundary and external force are considered. As usual, the main estimate is to obtain the lower bound on the density function. When the gas connects to vacuum continuously, the viscosity coefficient also vanishes at the boundary. In this case, we can expect only a lower bound of the density function in the form of a power function $x^{\beta}$ for some constant $\beta$ determined by the initial data and viscosity coefficient.

Furthermore, the interface separating the gas and vacuum propagates with finite speed because of the hyperbolic-parabolic property of the system (1.1). And the proof of this finite speed propagation follows from the lower bound of the density function estimate.

Some of the previous works in this direction can be summarized as follows. When the viscosity coefficient $\mu$ is a constant, there is no continuous dependence on the initial data to the Navier-Stokes equations (1.1) with vacuum, see [8]. This leads to the study on the initial boundary value problem instead of initial value problem. For this, the free boundary problem of one dimensional Navier-Stokes equations with one boundary fixed and the other connected to vacuum was investigated in [19], where the global existence of the weak solutions was proved. Similar results were obtained in [20] for the equations of spherically symmetric motion of viscous gases. Furthermore, the free boundary problem of the one-dimensional viscous gas expanding into the vacuum has been studied by many people, see $[19,20,25]$ and reference therein. A further understanding of the regularity and behavior of solutions near the interfaces between the gas and vacuum was given in [15].

It is known that the physically important case related to vacuum is when $\mu$ is not a constant, see $[23,24,31]$. It can be seen from the derivation of the Navier-Stokes equations from the Boltzmann equation through the Chapman-Enskog expansion to the second order, cf. [5], where the viscosity coefficient depends on the temperature. For isentropic flow, this dependence is reduced to the dependence on the density by the laws of Boyle and Gay-Lussac for ideal gas as discussed in [14]. When the viscosity coefficient is a function 
of density, such as $\mu=c \rho^{\theta}$ with $c$ and $\theta$ being positive constants, the local existence of weak solutions to Navier-Stokes equations with vacuum was studied in [14] on the case when the initial density connects to vacuum with discontinuities. The global existence of weak solutions was obtained in [21] with the same assumption when $0<\theta<\frac{1}{3}$ and it is later generalized to the cases for $0<\theta<\frac{1}{2}$ and $0<\theta<1$ in [28] and [11] respectively.

It is noticed that the above analysis is based on the uniform positive lower bound of the density with respect to the construction of the approximate solutions. This estimate is crucial because the other estimates for the convergence of a subsequence of the approximate solutions and the uniqueness of the solution thus obtained will follow from the estimation by standard techniques as long as the vacuum does not appear in the solutions in finite time. And this uniform positive lower bound on the density function can only be obtained when the density function connects to vacuum with discontinuities. In this situation, the density function is positive for any finite time and thus the viscosity coefficient never vanishes. This good property of the solution was obtained and used to prove global existence of solutions to (1.1) when the initial data is of compact support, cf. [11, 21, 28].

If the density function connects to vacuum continuously, there is no positive lower bound for the density function and the viscosity coefficient vanishes at vacuum. This degeneracy in the viscosity coefficient gives arise to new analysis difficulties because of the less regularizing effect on the solutions. A local existence result was obtained on this case in [29], and global existence result in [30] for $0<\theta<\frac{2}{9}$ and in [26] for $0<\theta<\frac{1}{3}$. Another difficulty comes from the singularity at the vacuum boundary when the density function connects to vacuum continuously. This can be seen from the analysis in [25] on the non-global existence of regular solution to Navier-Stokes equations when the density function is of compact support with the viscosity coefficient being constant. The proof there is based on the estimation on the growth rate of the support on density function in time $t$. If the growth rate is sub-linear, then a nonlinear functional was introduced in [25] which yields the non-global existence of regular solutions. The intuitive explanation of this phenomena comes from the consideration of the pressure in the gas. No matter how smooth the initial data is, the pressure of the gas will build up at the vacuum boundary in finite time and it will push the gas into the vacuum region. This effect can not be compensated by the dissipation from the viscosity so that the support of the gas stays unchanged. This is different from the system of Euler-Poisson equations for gaseous stars where the pressure and the gravitational force can become balanced to have a stationary solutions. In the case of compressible Navier-Stokes equation, the pressure will have the effort on the evolution of the vacuum boundary in finite time so that the density function at the interface will not be smooth. This singularity at the derivatives, maybe of the second order for one-dimensional case, cf. [25], gives some analytic difficulty, but it can be overcome by introducing some appropriate weights in the energy estimates as in $[30,26,4]$. Notice that these weight functions vanish at the vacuum boundary.

The last but not least, there has been a lot of investigation on the Navier-Stokes equations when the initial density is away from vacuum, both for smooth initial data or discontinuous initial data, and one dimensional or multidimensional problems. For these 
results, please refer to $[6,8,10,12,13,22]$ and reference therein. The non-appearance of vacuum in the solutions for any finite time if the initial data does not contain vacuum was proved in [9]. It is not known yet whether the corresponding result holds for the density-dependent viscosity.

The rest of this paper is organized as follows. In Section 2, we will give the definition of the weak solution and then state the main theorems in this paper. In Section 3, we will prove some a priori estimates for the global existence of weak solutions to (1.1)-(1.3). In Section 4, we will construct the approximate solutions by the line method and state a series of lemmas following the estimates on Section 3. A uniqueness theorem for the weak solution will be given in the last section.

\section{The Main Theorems}

The free boundary value problem (1.1)-(1.4) can be reformed in Lagrangian coordinates by using the transformation:

$$
x=\int_{0}^{\xi} \rho(z, \tau) d z, \quad t=\tau, \text { i.e., } \frac{\partial}{\partial \tau}=\frac{\partial}{\partial t}-\rho u \frac{\partial}{\partial x}, \quad \frac{\partial}{\partial \xi}=\rho \frac{\partial}{\partial x},
$$

Now, considering the position of boundary $X(\tau)=x(y(\tau), \tau)=\int_{0}^{y(\tau)} \rho(z, \tau) d z$ and using $(1.1)_{1},(1.3)$ and (1.4), we have $\frac{d X}{d \tau}=0$, i.e., $X$ is independent of $\tau$. Thus we can set $X \equiv \int_{0}^{y(0)} \rho(z, 0) d z$. We consider $y(0)>-\infty$ and $X>-\infty$, i.e., a finite total mass on a finite interval. After rescaling the variables, the problem (1.1)-(1.4) is transformed to the following fixed boundary problem:

$$
\left\{\begin{array}{l}
\rho_{t}+\rho^{2} u_{x}=0, \\
u_{t}+P(\rho)_{x}=\left(\mu \rho u_{x}\right)_{x}+g, \quad 0<x<1, \quad t>0,
\end{array}\right.
$$

with the boundary conditions

$$
\left\{\begin{array}{l}
\rho(0, t)=0 \text { at the free end, } \\
u(1, t)=0 \text { at the fixed end }
\end{array}\right.
$$

the initial data

$$
(\rho, u)(x, 0)=\left(\rho_{0}(x), u_{0}(x)\right), \quad 0 \leq x \leq 1,
$$

and the compatibility conditions at $x=0,1$,

$$
\rho_{0}(0)=0, \quad u_{0}(1)=0,
$$

where $P(\rho)=A \rho^{\gamma}, \mu=c \rho^{\theta}$. We normalize $A=1$ and $c=1$.

Throughout this paper, the assumptions on the initial data, $\theta$ and $\gamma$ can be stated as follows:

(A1) $0<\theta<\frac{1}{3}, \quad \gamma>1$ 
(A2) There exists a positive constant $C$ such that $0 \leq \rho_{0}(x) \leq C$ with $\rho_{0}(0)=0$, $\rho_{0}(1)>0,\left(\rho_{0}(x)\right)^{-1} \in L^{1}([0,1]),\left(\rho_{0}^{\gamma}(x)\right)_{x} \in L^{2}([0,1]), u_{0}(x) \in L^{\infty}([0,1])$ and $\left(\rho_{0}^{1+\theta}(x) u_{0 x}(x)\right)_{x} \in$ $L^{2}([0,1])$;

(A3) For $0<\theta<\frac{1}{3}$, there exists a sufficiently large positive integer $m$ such that

$$
\frac{2 m-1}{4 m^{2}} \leq \theta \leq \frac{4 m^{2}-8 m+3}{12 m^{2}-14 m+2}
$$

For such fixed positive integer $m$, let $x^{2 m-2}\left[\left(\rho_{0}^{\theta}\right)_{x}\right]^{2 m} \in L^{1}([0,1])$.

Under the assumptions (A1)-(A3), we will prove the existence of global weak solutions to the initial boundary value problem (2.1)-(2.4). The weak solution defined bellow is similar to the one in [21].

Definition 2.1. A pair of functions $(\rho(x, t), u(x, t))$ is called a global weak solution to the initial boundary value problem (2.1)-(2.4), if for any $T>0$

$$
\begin{gathered}
\rho, u \in L^{\infty}([0,1] \times[0, T]) \cap C^{1}\left([0, T] ; L^{2}([0,1])\right), \\
\rho^{1+\theta} u_{x} \in L^{\infty}([0,1] \times[0, T]) \cap C^{\frac{1}{2}}\left([0, T] ; L^{2}([0,1])\right) .
\end{gathered}
$$

Furthermore, the following equations hold:

$$
\int_{0}^{\infty} \int_{0}^{1}\left(\rho \phi_{t}-\rho^{2} u_{x} \phi\right) d x d t+\int_{0}^{1} \rho_{0}(x) \phi(x, 0) d x=0,
$$

and

$$
\int_{0}^{\infty} \int_{0}^{1}\left(u \psi_{t}+\left(P(\rho)-\mu \rho u_{x}\right) \psi_{x}-g \psi\right) d x d t+\int_{0}^{1} u_{0}(x) \psi(x, 0) d x=0,
$$

for any test functions $\phi(x, t)$ and $\psi(x, t) \in C_{0}^{\infty}(\Omega)$ with $\Omega=\{(x, t): 0 \leq x \leq 1, t \geq 0\}$.

In what follows, we always use $C(C(T))$ to denote a generic positive constant depending only on the initial data (or the given time $T$ ).

We now state the main theorem in this paper as follows:

Theorem 2.2 (Existence). Under the conditions (A1)-(A3), the free boundary problem (2.1)-(2.4) has a weak solution $(\rho(x, t), u(x, t))$ which satisfies Definition 2.1 and $\rho(x, t)$ satisfies

$$
C(T) x^{1+k_{3}} \leq \rho(x, t) \leq C(T)
$$

where $k_{3}$ satisfies

$$
\frac{2 m \theta}{2 m(1-\theta)-1}=\max \left\{\frac{1}{2 m-1}, \frac{2 m \theta}{2 m(1-\theta)-1}\right\} \leq k_{3} \leq \frac{1}{2 \theta}-1-\frac{1}{(2 m-1) \theta} .
$$


Remark 2.3. $\quad$ There exists some constant $k_{3}$ which satisfies (2.11).

In fact, from (2.5), we have

$$
\frac{1}{2 m-1} \leq \frac{2 m \theta}{2 m(1-\theta)-1} \leq \frac{1}{2 \theta}-1-\frac{1}{(2 m-1) \theta}
$$

Choose

$$
k_{3}=\frac{1}{2}\left(\frac{2 m \theta}{2 m(1-\theta)-1}+\frac{1}{2 \theta}-1-\frac{1}{(2 m-1) \theta}\right),
$$

so that $k_{3}$ satisfies $(2.11)$.

To get the uniqueness of the weak solution, we need the following assumptions:

(A1)' For any $0<\alpha<1$, let $0<\theta<\frac{\alpha}{1+2 \alpha+\sqrt{1+4 \alpha+\alpha^{2}}}$, and $\gamma>1$;

(A2) There exists a positive constant $C$ such that $0 \leq \rho_{0}(x) \leq C x^{\alpha}$ with $0<\alpha<1$, $\rho_{0}(0)=0, \rho_{0}(1)>0,\left(\rho_{0}(x)\right)^{-1} \in L^{1}([0,1]),\left(\rho_{0}^{\gamma}(x)\right)_{x} \in L^{2}([0,1]), u_{0}(x) \in L^{\infty}([0,1])$ and $\left(\rho_{0}^{1+\theta}(x) u_{0 x}(x)\right)_{x} \in L^{2}([0,1])$

(A3) $)^{\prime}$ For $0<\theta<\frac{\alpha}{1+2 \alpha+\sqrt{1+4 \alpha+\alpha^{2}}}$, there exists a sufficiently large positive integer $m$ such that

$$
\left\{\begin{array}{l}
4 \theta m^{2}-2 m+1 \geq 0 \\
4(1-3 \theta) m^{2}-(8-14 \theta) m+(3-2 \theta) \geq 0 \\
4\left(3 \alpha \theta^{2}-(4 \alpha+2) \theta+\alpha\right) m^{2}-4\left(\alpha \theta^{2}-(3 \alpha+2) \theta+\alpha\right) m+\alpha-(2 \alpha+2) \theta \geq 0 .
\end{array}\right.
$$

For such fixed positive integer $m$, let $x^{2 m-2}\left[\left(\rho_{0}^{\theta}\right)_{x}\right]^{2 m} \in L^{1}([0,1])$.

It is noted that assumptions (A1)', (A2)' and (A3)' imply assumptions (A1), (A2) and (A3), respectively. Then the uniqueness of the weak solution can be stated as follows:

Theorem 2.4 (Uniqueness). Assume $(A 1)^{\prime}-(A 3)^{\prime}$, let $\left(\rho_{1}, u_{1}\right)(x, t)$ and $\left(\rho_{2}, u_{2}\right)(x, t)$ be two weak solutions to the initial boundary value problem (2.1)-(2.4) in $0 \leq t \leq T$ as described in Definition 2.1. Then

$$
\left(\rho_{1}, u_{1}\right)(x, t)=\left(\rho_{2}, u_{2}\right)(x, t)
$$

for a.e. $(x, t) \in[0,1] \times[0, T]$.

\section{Some a priori estimates}

In this section, for simplicity of presentation, we establish a series of a priori estimates in continuous version to the initial boundary value problem (2.1)-(2.4). The corresponding discrete version will be given in Section 4 . 
Lemma 3.1. Under the conditions of Theorem 2.2, we have that for $0<x<1, t>0$,

$$
\begin{gathered}
\left(\rho^{\theta}\right)_{t}(x, t)=-\theta \rho^{1+\theta} u_{x}(x, t) \\
\left(\rho^{1+\theta} u_{x}\right)(x, t)=\rho^{\gamma}(x, t)+\int_{0}^{x} u_{t}(y, t) d y-g x
\end{gathered}
$$

and

$$
\rho^{\theta}(x, t)+\theta \int_{0}^{t} \rho^{\gamma}(x, s) d s=\rho_{0}^{\theta}(x)+\theta g x t-\theta \int_{0}^{t} \int_{0}^{x} u_{t}(y, s) d y d s .
$$

Proof. From $(2.1)_{1}$, we have

$$
\left(\rho^{\theta}\right)_{t}=\theta \rho^{\theta-1} \rho_{t}=-\theta \rho^{1+\theta} u_{x}
$$

which implies (3.1).

Integrating $(2.1)_{2}$ over $[0, x]$ and using the boundary condition (2.2), we have

$$
\int_{0}^{x} u_{t}(y, t) d y+\rho^{\gamma}(x, t)=\left(\rho^{1+\theta} u_{x}\right)(x, t)+g x
$$

which implies (3.2).

Integrating (3.1) over $[0, t]$, we have

$$
\rho^{\theta}(x, t)=-\theta \int_{0}^{t}\left(\rho^{1+\theta} u_{x}\right)(x, s) d s+\rho_{0}^{\theta}(x)
$$

From (3.2) and (3.4), we get (3.3). Lemma 3.1 is completed.

Lemma 3.2. Under the conditions of Theorem 2.2, the following energy estimates hold:

$$
\begin{aligned}
& \int_{0}^{1}\left(\frac{1}{2} u^{2}+\frac{1}{\gamma-1} \rho^{\gamma-1}+\frac{g x}{\rho}\right) d x+\int_{0}^{t} \int_{0}^{1} \rho^{1+\theta} u_{x}^{2} d x d t \\
= & \int_{0}^{1}\left(\frac{1}{2} u_{0}^{2}(x)+\frac{1}{\gamma-1} \rho_{0}^{\gamma-1}(x)+\frac{g x}{\rho_{0}(x)}\right) d x \\
\leq & C, \quad 0<t \leq T .
\end{aligned}
$$

Proof. Multiplying $(2.1)_{1}$ and $(2.2)_{2}$ by $\rho^{\gamma-2}-g x \rho^{-2}$ and $u$, respectively and summing them, we get

$$
\frac{d}{d t}\left(\frac{1}{2} u^{2}+\frac{1}{\gamma-1} \rho^{\gamma-1}+\frac{g x}{\rho}\right)+\left(\rho^{\gamma} u\right)_{x}-g x u_{x}=u\left(\rho^{1+\theta} u_{x}\right)_{x}+g u
$$


Integrating (3.6) over $[0,1] \times[0, t]$ and using the boundary condition $(2.2)$ and the assumption (A1) and (A2), we have

$$
\begin{aligned}
& \int_{0}^{1}\left(\frac{1}{2} u^{2}+\frac{1}{\gamma-1} \rho^{\gamma-1}+\frac{g x}{\rho}\right) d x+\int_{0}^{t} \int_{0}^{1} \rho^{1+\theta} u_{x}^{2} d x d t \\
= & \int_{0}^{1}\left(\frac{1}{2} u_{0}^{2}(x)+\frac{1}{\gamma-1} \rho_{0}^{\gamma-1}(x)+\frac{g x}{\rho_{0}(x)}\right) d x+\int_{0}^{t} \int_{0}^{1} g u d x d s+\int_{0}^{t} \int_{0}^{1} g x u_{x} d x d s \\
= & \int_{0}^{1}\left(\frac{1}{2} u_{0}^{2}(x)+\frac{1}{\gamma-1} \rho_{0}^{\gamma-1}(x)+\frac{g x}{\rho_{0}(x)}\right) d x+\left.\int_{0}^{t}(g x u)\right|_{x=0} ^{x=1} d s \\
= & \int_{0}^{1}\left(\frac{1}{2} u_{0}^{2}(x)+\frac{1}{\gamma-1} \rho_{0}^{\gamma-1}(x)+\frac{g x}{\rho_{0}(x)}\right) d x \\
\leq & \int_{0}^{1}\left(\frac{1}{2} u_{0}^{2}(x)+\frac{1}{\gamma-1} \rho_{0}^{\gamma-1}(x)+\frac{g}{\rho_{0}(x)}\right) d x,
\end{aligned}
$$

which implies (3.5). The proof of Lemma 3.2 is completed.

Lemma 3.3. $\quad$ Under the conditions of Theorem 2.2, we have

$$
\rho(x, t) \leq C(T), \quad 0<x<1, \quad 0<t \leq T .
$$

Proof. Using (3.3), Cauchy-Schwarz inequality, the assumption (A2) and Lemma 3.2, we have that for $0<x<1,0<t \leq T$,

$$
\begin{aligned}
\rho^{\theta}(x, t)+\theta \int_{0}^{t} \rho^{\gamma}(x, s) d s & =\rho_{0}^{\theta}(x)+\theta g x t-\theta \int_{0}^{t} \int_{0}^{x} u_{t}(y, s) d y d s \\
& =\rho_{0}^{\theta}(x)+\theta g x t+\theta \int_{0}^{x} u_{0}(y) d y-\theta \int_{0}^{x} u(y, t) d y \\
& \leq C(T)+C\left\|u_{0}\right\|_{L^{\infty}([0,1])}+C \int_{0}^{1} u^{2} d x \\
& \leq C(T),
\end{aligned}
$$

which implies (3.8). Lemma 3.3 is completed.

Lemma 3.4. For the positive integer $m$ defined by (2.5), we have

$$
\int_{0}^{1} u^{2 m} d x+\int_{0}^{t} \int_{0}^{1} u^{2 m-2} \rho^{1+\theta} u_{x}^{2} d x d s \leq C(T)
$$

Proof. Multiplying $(2.1)_{2}$ by $2 m u^{2 m-1}$, integrating it over $[0,1] \times[0, t]$ and using the boundary condition (2.2), we have

$$
\begin{aligned}
& \int_{0}^{1} u^{2 m} d x+2 m(2 m-1) \int_{0}^{t} \int_{0}^{1} u^{2 m-2} \rho^{1+\theta} u_{x}^{2} d x d s \\
& =\int_{0}^{1} u_{0}^{2 m} d x+2 m(2 m-1) \int_{0}^{t} \int_{0}^{1} u^{2 m-2} \rho^{\gamma} u_{x} d x d s+2 m g \int_{0}^{t} \int_{0}^{1} u^{2 m-1} d x d s .
\end{aligned}
$$


Using the assumption (A2), Cauchy-Schwarz inequality and Young's inequality $a b \leq \frac{1}{p} a^{p}+$ $\frac{1}{q} b^{q}$ for $a, b \geq 0, p, q>1, \frac{1}{p}+\frac{1}{q}=1$, we have

$$
\begin{aligned}
& \int_{0}^{1} u^{2 m} d x+2 m(2 m-1) \int_{0}^{t} \int_{0}^{1} u^{2 m-2} \rho^{1+\theta} u_{x}^{2} d x d s \\
\leq & C+m(2 m-1) \int_{0}^{t} \int_{0}^{1} u^{2 m-2} \rho^{1+\theta} u_{x}^{2} d x d s+m(2 m-1) \int_{0}^{t} \int_{0}^{1} u^{2 m-2} \rho^{2 \gamma-1-\theta} d x d s \\
& +(2 m-1) \int_{0}^{t} \int_{0}^{1} u^{2 m} d x d s+\int_{0}^{t} \int_{0}^{1} g^{2 m} d x d s \\
\leq & C(T)+m(2 m-1) \int_{0}^{t} \int_{0}^{1} u^{2 m-2} \rho^{1+\theta} u_{x}^{2} d x d s \\
& +m(2 m-1) \int_{0}^{t} \int_{0}^{1}\left(\frac{1}{m} \rho^{(2 \gamma-1-\theta) m}+\frac{m-1}{m} u^{2 m}\right) d x d s+(2 m-1) \int_{0}^{t} \int_{0}^{1} u^{2 m} d x d s
\end{aligned}
$$

which implies together with Lemma 3.3 and $2 \gamma-1-\theta>0$ that

$$
\begin{aligned}
& \int_{0}^{1} u^{2 m} d x+m(2 m-1) \int_{0}^{t} \int_{0}^{1} u^{2 m-2} \rho^{1+\theta} u_{x}^{2} d x d s \\
\leq & C(T)+m(2 m-1) \int_{0}^{t} \int_{0}^{1} u^{2 m} d x d s .
\end{aligned}
$$

Then (3.10) shows

$$
\int_{0}^{1} u^{2 m} d x \leq C(T)+m(2 m-1) \int_{0}^{t} \int_{0}^{1} u^{2 m} d x d s
$$

By Gronwall's inequality, we have

$$
\int_{0}^{1} u^{2 m} d x \leq C(T) e^{m(2 m-1) t}
$$

Substituting (3.11) into the right-hand of (3.10), we deduce (3.9). The proof of Lemma 3.4 is completed.

Now we will prove a weighted energy estimate on the function $\left(\rho^{\theta}\right)_{x}$.

Lemma 3.5. Under the conditions of Theorem 2.2, we have for the positive integer $m$ defined by (2.5) and any positive constant $k_{1} \geq 2 m-2$ that

$$
\int_{0}^{1} x^{k_{1}}\left[\left(\rho^{\theta}\right)_{x}\right]^{2 m} d x \leq C(T)
$$

Proof. From (3.1), we have

$$
\left(\rho^{\theta}\right)_{t}=-\theta \rho^{1+\theta} u_{x}
$$


which implies by using $(2.1)_{2}$

$$
\left(\rho^{\theta}\right)_{x t}=-\theta\left(\rho^{1+\theta} u_{x}\right)_{x}=-\theta\left(u_{t}+\left(\rho^{\gamma}\right)_{x}\right)+\theta g
$$

Integrating (3.14) with respect to $t$ over $[0, t]$, we have

$$
\left(\rho^{\theta}\right)_{x}=\left(\rho_{0}^{\theta}\right)_{x}-\theta\left(u(x, t)-u_{0}(x)\right)-\theta \int_{0}^{t}\left(\rho^{\gamma}\right)_{x} d s+\theta g t
$$

Multiplying (3.15) by $x^{k_{1}}\left[\left(\rho^{\theta}\right)_{x}\right]^{2 m-1}$ and integrating it with respect to $x$ over $[0,1]$, we have

$$
\begin{aligned}
\int_{0}^{1} x^{k_{1}}\left[\left(\rho^{\theta}\right)_{x}\right]^{2 m} d x= & \int_{0}^{1} x^{k_{1}}\left(\rho_{0}^{\theta}\right)_{x}\left[\left(\rho^{\theta}\right)_{x}\right]^{2 m-1} d x \\
& -\theta \int_{0}^{1} x^{k_{1}}\left(u(x, t)-u_{0}(x)\right)\left[\left(\rho^{\theta}\right)_{x}\right]^{2 m-1} d x \\
& -\theta \int_{0}^{1} x^{k_{1}}\left[\left(\rho^{\theta}\right)_{x}\right]^{2 m-1} \int_{0}^{t}\left(\rho^{\gamma}\right)_{x} d s d x \\
& +\theta g t \int_{0}^{1} x^{k_{1}}\left[\left(\rho^{\theta}\right)_{x}\right]^{2 m-1} d x
\end{aligned}
$$

Using Young's inequality $a b \leq \varepsilon a^{p}+C(\varepsilon) b^{q}$ for $a, b \geq 0, \varepsilon>0, C(\varepsilon)=(\varepsilon p)^{-\frac{q}{p}} q^{-1}$, we have

$$
\begin{aligned}
& \int_{0}^{1} x^{k_{1}}\left[\left(\rho^{\theta}\right)_{x}\right]^{2 m} d x \\
\leq & \frac{1}{8} \int_{0}^{1} x^{k_{1}}\left[\left(\rho^{\theta}\right)_{x}\right]^{2 m} d x+C \int_{0}^{1} x^{k_{1}}\left[\left(\rho_{0}^{\theta}\right)_{x}\right]^{2 m} d x \\
& +\frac{1}{8} \int_{0}^{1} x^{k_{1}}\left[\left(\rho^{\theta}\right)_{x}\right]^{2 m} d x+C \int_{0}^{1} x^{k_{1}}\left(u^{2 m}+u_{0}^{2 m}\right) d x \\
& +\frac{1}{8} \int_{0}^{1} x^{k_{1}}\left[\left(\rho^{\theta}\right)_{x}\right]^{2 m} d x+C \int_{0}^{1} x^{k_{1}}\left(\int_{0}^{t}\left|\left(\rho^{\gamma}\right)_{x}\right| d s\right)^{2 m} d x \\
& +\frac{1}{8} \int_{0}^{1} x^{k_{1}}\left[\left(\rho^{\theta}\right)_{x}\right]^{2 m} d x+C t^{2 m} \int_{0}^{1} x^{k_{1}} d x \\
\leq & \frac{1}{2} \int_{0}^{1} x^{k_{1}}\left[\left(\rho^{\theta}\right)_{x}\right]^{2 m} d x+C \int_{0}^{1} x^{k_{1}}\left(\int_{0}^{t}\left|\left(\rho^{\gamma}\right)_{x}\right| d s\right)^{2 m} d x \\
& +C \max _{[0,1]}\left(x^{k_{1}-(2 m-2)}\right) \int_{0}^{1} x^{2 m-2}\left[\left(\rho_{0}^{\theta}\right)_{x}\right]^{2 m} d x+C \int_{0}^{1}\left(u^{2 m}+u_{0}^{2 m}\right) d x+C(T),
\end{aligned}
$$

Noticing $k_{1} \geq 2 m-2$ and using Lemma 3.3, Lemma 3.4, assumptions (A2), (A3) and 
Hölder's inequality, we have from (3.17)

$$
\begin{aligned}
\int_{0}^{1} x^{k_{1}}\left[\left(\rho^{\theta}\right)_{x}\right]^{2 m} d x & \leq C(T) \int_{0}^{1} x^{k_{1}} \int_{0}^{t}\left[\left(\rho^{\gamma}\right)_{x}\right]^{2 m} d s d x+C(T) \\
& \leq C(T) \int_{0}^{t} \max _{[0,1]}\left(\rho^{\gamma-\theta}\right)^{2 m} \int_{0}^{1} x^{k_{1}}\left[\left(\rho^{\theta}\right)_{x}\right]^{2 m} d x d s+C(T) \\
& \leq C(T) \int_{0}^{t} \int_{0}^{1} x^{k_{1}}\left[\left(\rho^{\theta}\right)_{x}\right]^{2 m} d x d s+C(T)
\end{aligned}
$$

Gronwall inequality implies Lemma 3.5.

For the positive integer $m$ defined by (2.5), if we choose $k_{1}=2 m-2$, then we have the following result:

Corollary 3.6. Under the conditions of Theorem 2.2, we have

$$
\int_{0}^{1} x^{2 m-2}\left[\left(\rho^{\theta}\right)_{x}\right]^{2 m} d x \leq C(T) .
$$

Based on Lemma 3.3, Lemma 3.4 and Corollary 3.6, the following lemma gives this kind of estimate with a weighted function $x^{k_{2}}$.

Lemma 3.7. For any $k_{2}>\frac{1}{2 m}$, we have

$$
\int_{0}^{1} \frac{x^{k_{2}}}{\rho(x, t)} d x \leq C(T)
$$

Proof. $\quad$ From $(2.1)_{1}$, we have

$$
\left(\frac{x^{k_{2}}}{\rho(x, t)}\right)_{t}=x^{k_{2}} u_{x}(x, t) .
$$

Integrating $(3.20)$ over $[0,1] \times[0, T]$ and using Young's inequality, we have

$$
\begin{aligned}
\int_{0}^{1} \frac{x^{k_{2}}}{\rho(x, t)} d x & =\int_{0}^{1} \frac{x^{k_{2}}}{\rho_{0}(x)} d x+\int_{0}^{t} \int_{0}^{1} x^{k_{2}} u_{x}(x, s) d x d s \\
& =\int_{0}^{1} \frac{x^{k_{2}}}{\rho_{0}(x)} d x+\left.\int_{0}^{t}\left(x^{k_{2}} u(x, s)\right)\right|_{x=0} ^{x=1} d s-k_{2} \int_{0}^{t} \int_{0}^{1} x^{k_{2}-1} u(x, s) d x d s \\
& \leq C+C \int_{0}^{t} \int_{0}^{1} u^{2 m}(x, s) d x d s+C \int_{0}^{t} \int_{0}^{1} x^{\frac{2 m\left(k_{2}-1\right)}{2 m-1}} d x d s
\end{aligned}
$$

By using Lemma 3.4 and noticing $\frac{2 m\left(k_{2}-1\right)}{2 m-1}>-1$ when $k_{2}>\frac{1}{2 m}$, we have

$$
\int_{0}^{1} \frac{x^{k_{2}}}{\rho(x, t)} d x \leq C(T)
$$

This proves Lemma 3.7. 
Remark 3.8. The finite propagation property implies that the finiteness of the integral $\int_{0}^{1} \frac{1}{\rho(x, t)}$ which is stronger than Lemma 3.7. However, this boundedness can not be obtained here without using a weight $x^{k_{2}}$, where $k_{2}$ is a positive constant which can be arbitrarily small. The boundedness of $\int_{0}^{1} \frac{1}{\rho(x, t)}$ holds once the $L^{\infty}$ bound on the velocity is given in Lemma 3.13.

If we choose $k_{2}=\frac{1}{2 m-1}\left(>\frac{1}{2 m}\right)$ in Lemma 3.7, then we have the following result which is used to get the lower bound estimate of the density function $\rho(x, t)$.

Corollary 3.9. The following estimate holds:

$$
\int_{0}^{1} \frac{x^{\frac{1}{2 m-1}}}{\rho(x, t)} d x \leq C(T),
$$

where $m$ is defined by (2.5).

The next lemma gives a estimate on the lower bound for the density function $\rho(x, t)$. This crucial estimate can be used to study the other property of the solution $(\rho, u)(x, t)$ for compactness of the sequence of the approximate solutions given in the next section.

Lemma 3.10. For any $0<\theta<\frac{1}{3}$, the following estimate holds

$$
\rho(x, t) \geq C(T) x^{1+k_{3}},
$$

where $k_{3}$ satisfies (2.11).

Proof. Now by using Sobolev's embedding theorem $W^{1,1}([0,1]) \hookrightarrow L^{\infty}([0,1])$ and Hölder's inequality, we have from Corollary 3.9 and Lemma 3.5

$$
\begin{aligned}
\frac{x^{1+k_{3}}}{\rho(x, t)} \leq & \int_{0}^{1} \frac{x^{1+k_{3}}}{\rho(x, t)} d x+\int_{0}^{1}\left|\left(\frac{x^{1+k_{3}}}{\rho(x, t)}\right)_{x}\right| d x \\
\leq & \max _{[0,1]}\left(x^{1+k_{3}-k_{2}}\right) \int_{0}^{1} \frac{x^{k_{2}}}{\rho(x, t)} d x+\int_{0}^{1} \frac{x^{1+k_{3}}\left|\rho_{x}(x, t)\right|}{\rho^{2}(x, t)} d x \\
& +\left(1+k_{3}\right) \max _{[0,1]}\left(x^{k_{3}-k_{2}}\right) \int_{0}^{1} \frac{x^{k_{2}}}{\rho(x, t)} d x \\
\leq & C(T)+\frac{1}{\theta} \int_{0}^{1} \frac{x^{1+k_{3}}\left|\left(\rho^{\theta}(x, t)\right)_{x}\right|}{\rho^{1+\theta}(x, t)} d x \\
\leq & C(T)+\frac{1}{\theta}\left(\int_{0}^{1} x^{k_{1}}\left[\left(\rho^{\theta}\right)_{x}\right]^{2 m} d x\right)^{\frac{1}{2 m}}\left(\int_{0}^{1} x^{\left(1+k_{3}-\frac{k_{1}}{2 m}\right) q} \rho^{-(1+\theta) q} d x\right)^{\frac{1}{q}} \\
\leq & C(T)+C(T)\left(\int_{0}^{1} \frac{x^{k_{2}}}{\rho(x, t)} d x\right)^{\frac{1}{q}} \max _{[0,1]}\left(\frac{x^{\left(1+k_{3}-\frac{k_{1}}{2 m}\right) q-k_{2}}}{\rho^{(1+\theta) q-1}}\right)^{\frac{1}{q}} \\
\leq & C(T)+C(T) \max _{[0,1]}\left(\frac{x^{1+k_{3}}}{\rho(x, t)}\right)^{1+\theta-\frac{1}{q}} \max _{[0,1]} x^{k_{4}},
\end{aligned}
$$


where $q=\frac{2 m}{2 m-1}$ and $k_{4}=1+k_{3}-\frac{k_{1}}{2 m}-\frac{k_{2}}{q}-\left(1+k_{3}\right)\left(1+\theta-\frac{1}{q}\right)$. Here we have used $k_{3} \geq \frac{1}{2 m-1}=k_{2}$ from $(2.11)$.

By $k_{3} \geq \frac{2 m \theta}{2 m(1-\theta)-1}$, we have

$$
k_{4}=1+k_{3}-\frac{k_{1}}{2 m}-\frac{k_{2}}{q}-\left(1+k_{3}\right)\left(1+\theta-\frac{1}{q}\right)=k_{3}\left(1-\theta-\frac{1}{2 m}\right)-\theta \geq 0 .
$$

This and (3.24) show

$$
\max _{[0,1]} \frac{x^{1+k_{3}}}{\rho(x, t)} \leq C(T)+C(T)\left(\max _{[0,1]} \frac{x^{1+k_{3}}}{\rho(x, t)}\right)^{1+\theta-\frac{1}{q}},
$$

i.e.,

$$
\max _{[0,1]} \frac{x^{1+k_{3}}}{\rho(x, t)} \leq C(T)+C(T)\left(\max _{[0,1]} \frac{x^{1+k_{3}}}{\rho(x, t)}\right)^{\theta+\frac{1}{2 m}} .
$$

For $0<\theta<\frac{1}{3}$, we have $0<\theta+\frac{1}{2 m}<1$. Therefore, (3.25) implies

$$
\max _{[0,1]} \frac{x^{1+k_{3}}}{\rho(x, t)} \leq C(T) .
$$

This proves (3.23) and the proof of Lemma 3.10 is completed.

Lemma 3.11. Under the conditions of Theorem 2.2, we have

$$
\int_{0}^{1} u_{t}^{2} d x+\int_{0}^{t} \int_{0}^{1} \rho^{1+\theta} u_{x t}^{2} d x d s \leq C(T)
$$

Proof. Differentiating $(2.1)_{2}$ with respect to $t$, multiplying it by $2 u_{t}$ and integrating it over $[0,1] \times[0, t]$, we get

$$
\int_{0}^{1} u_{t}^{2} d x+2 \int_{0}^{t} \int_{0}^{1}\left(\rho^{\gamma}\right)_{x t} u_{t} d x d s=\int_{0}^{1} u_{0 t}^{2} d x+2 \int_{0}^{t} \int_{0}^{1}\left(\rho^{1+\theta} u_{x}\right)_{x t} u_{t} d x d s .
$$

Since

$$
u_{0 t}=\left(\rho_{0}^{1+\theta} u_{0 x}\right)_{x}+g-\left(\rho_{0}^{\gamma}\right)_{x},
$$

we have from the assumption (A2) that

$$
\int_{0}^{1} u_{0 t}^{2}(x) d x \leq C .
$$

On the other hand, using integration by parts, we have from $(2.1)_{1}$

$$
\begin{aligned}
& 2 \int_{0}^{t} \int_{0}^{1}\left(\rho^{1+\theta} u_{x}\right)_{x t} u_{t} d x d s \\
= & 2 \int_{0}^{t} \int_{0}^{1}\left\{\left(\rho^{1+\theta} u_{x}\right)_{t} u_{t}\right\}_{x} d x d s-2 \int_{0}^{t} \int_{0}^{1}\left(\rho^{1+\theta} u_{x}\right)_{t} u_{x t} d x d s \\
= & -2 \int_{0}^{t} \int_{0}^{1} \rho^{1+\theta} u_{x t}^{2} d x d s+2(1+\theta) \int_{0}^{t} \int_{0}^{1} \rho^{2+\theta} u_{x}^{2} u_{x t} d x d s .
\end{aligned}
$$


Similarly, we have

$$
\begin{aligned}
& 2 \int_{0}^{t} \int_{0}^{1}\left(\rho^{\gamma}\right)_{x t} u_{t} d x d s \\
= & 2 \int_{0}^{t} \int_{0}^{1}\left\{\left(\rho^{\gamma}\right)_{t} u_{t}\right\}_{x} d x d s-2 \int_{0}^{t} \int_{0}^{1}\left(\rho^{\gamma}\right)_{t} u_{x t} d x d s \\
= & 2 \gamma \int_{0}^{t} \int_{0}^{1} \rho^{1+\gamma} u_{x} u_{x t} d x d s .
\end{aligned}
$$

Here in (3.30) and (3.31), we have used the boundary condition (2.2) and the equation $(2.1)_{1}$.

Substituting (3.29)-(3.31) into (3.27), we have

$$
\begin{aligned}
& \int_{0}^{1} u_{t}^{2} d x+2 \int_{0}^{t} \int_{0}^{1} \rho^{1+\theta} u_{x t}^{2} d x d s \\
\leq & C+2(1+\theta) \int_{0}^{t} \int_{0}^{1} \rho^{2+\theta} u_{x}^{2} u_{x t} d x d s-2 \gamma \int_{0}^{t} \int_{0}^{1} \rho^{1+\gamma} u_{x} u_{x t} d x d s .
\end{aligned}
$$

From Cauchy-Schwarz inequality, we have

$$
\begin{aligned}
& 2(1+\theta) \int_{0}^{t} \int_{0}^{1} \rho^{2+\theta} u_{x}^{2} u_{x t} d x d s \\
\leq & \frac{1}{2} \int_{0}^{t} \int_{0}^{1} \rho^{1+\theta} u_{x t}^{2} d x d s+2(1+\theta)^{2} \int_{0}^{t} \int_{0}^{1} \rho^{3+\theta} u_{x}^{4} d x d s,
\end{aligned}
$$

and

$$
\begin{aligned}
& -2 \gamma \int_{0}^{t} \int_{0}^{1} \rho^{1+\gamma} u_{x} u_{x t} d x d s \\
\leq & \frac{1}{2} \int_{0}^{t} \int_{0}^{1} \rho^{1+\theta} u_{x t}^{2} d x d s+2 \gamma^{2} \int_{0}^{t} \int_{0}^{1} \rho^{2 \gamma+1-\theta} u_{x}^{2} d x d s .
\end{aligned}
$$

Therefore,

$$
\begin{aligned}
& \int_{0}^{1} u_{t}^{2} d x+\int_{0}^{t} \int_{0}^{1} \rho^{1+\theta} u_{x t}^{2} d x d s \\
\leq & C+2(1+\theta)^{2} \int_{0}^{t} \int_{0}^{1} \rho^{3+\theta} u_{x}^{4} d x d s+2 \gamma^{2} \int_{0}^{t} \int_{0}^{1} \rho^{2 \gamma+1-\theta} u_{x}^{2} d x d s \\
= & C+2(1+\theta)^{2} I_{1}+2 \gamma^{2} I_{2} .
\end{aligned}
$$

Now we can estimate $I_{1}$ and $I_{2}$ as follows:

$$
I_{1}=\int_{0}^{t} \int_{0}^{1} \rho^{3+\theta} u_{x}^{4} d x d s \leq \int_{0}^{t} \max _{[0,1]}\left(\rho^{2} u_{x}^{2}\right)(\cdot, s) V(s) d s,
$$

where

$$
V(s)=\int_{0}^{1}\left(\rho^{1+\theta} u_{x}^{2}\right)(x, s) d x .
$$


On the other hand, from (3.2), Lemma 3.3 and Lemma 3.10, we have by Hölder's inequality

$$
\begin{aligned}
\rho^{2} u_{x}^{2} & =\rho^{-2 \theta}\left(\rho^{1+\theta} u_{x}\right)^{2} \\
& =\rho^{-2 \theta}\left(\int_{0}^{x} u_{t}(y, t) d y+\rho^{\gamma}-g x\right)^{2} \\
& \leq C \rho^{-2 \theta} x \int_{0}^{1} u_{t}^{2} d x+C \rho^{2(\gamma-\theta)}+C g^{2} x^{2} \rho^{-2 \theta} \\
& \leq C(T) x^{1-2 \theta\left(1+k_{3}\right)} \int_{0}^{1} u_{t}^{2} d x+C(T)+C(T) x^{2-2 \theta\left(1+k_{3}\right)}
\end{aligned}
$$

Since $k_{3} \leq \frac{1}{2 \theta}-1-\frac{1}{(2 m-1) \theta} \leq \frac{1}{2 \theta}-1$, we have

$$
1-2 \theta\left(1+k_{3}\right) \geq 0
$$

which implies

$$
\max _{[0,1]}\left(\rho^{2} u_{x}^{2}\right)(\cdot, t) \leq C(T) \int_{0}^{1} u_{t}^{2} d x+C(T)
$$

Therefore

$$
I_{1} \leq C(T) \int_{0}^{t} V(s) \int_{0}^{1} u_{t}^{2} d x d s+C(T) \int_{0}^{t} V(s) d s .
$$

Similarly, we have by Lemma 3.2 and Lemma 3.3

$$
I_{2}=\int_{0}^{t} \int_{0}^{1} \rho^{2 \gamma+1-\theta} u_{x}^{2} d x d s=\max \left(\rho^{2 \gamma-2 \theta}\right) \int_{0}^{t} \int_{0}^{1} \rho^{1+\theta} u_{x}^{2} d x d s \leq C(T) .
$$

Substituting (3.38) and (3.39) into (3.35), we have by Lemma 3.2

$$
\int_{0}^{1} u_{t}^{2} d x+\int_{0}^{t} \int_{0}^{1} \rho^{1+\theta} u_{x t}^{2} d x d s \leq C(T)\left(1+\int_{0}^{t} V(s) \int_{0}^{1} u_{t}^{2} d x d s\right) .
$$

Gronwall's inequality and Lemma 3.2 give

$$
\int_{0}^{1} u_{t}^{2} d x \leq C(T) \exp \left(C(T) \int_{0}^{t} V(s) d s\right) \leq C(T) .
$$

Combining (3.40) with (3.41) and using Lemma 3.2, we can get (3.26) immediately. This completes the proof of Lemma 3.11.

Lemma 3.12. Under the conditions of Theorem 2.2, we have

$$
\begin{gathered}
\int_{0}^{1}\left|\rho_{x}(x, t)\right| d x \leq C(T), \\
\left\|\rho^{1+\theta}(x, t) u_{x}(x, t)\right\|_{L^{\infty}([0,1] \times[0, T])} \leq C(T),
\end{gathered}
$$

and

$$
\int_{0}^{1}\left|\left(\rho^{1+\theta} u_{x}\right)_{x}(x, t)\right| d x \leq C(T) .
$$


Proof. Since

$$
\left(\rho^{1+\theta} u_{x}\right)(x, t)=\int_{0}^{x} u_{t}(y, t) d y+\rho^{\gamma}(x, t)-g x,
$$

(3.43) follows from Lemma 3.3 and Lemma 3.11.

On the other hand, from $(2.1)_{2}$, we have by Lemma 3.11, Corollary 3.6 and Young's inequality

$$
\begin{aligned}
\int_{0}^{1}\left|\left(\rho^{1+\theta} u_{x}\right)_{x}(x, t)\right| d x & \leq \int_{0}^{1}\left|u_{t}(x, t)\right| d x+\int_{0}^{1}\left|\left(\rho^{\gamma}\right)_{x}(x, t)\right| d x+g \\
& \leq \int_{0}^{1} u_{t}^{2}(x, t) d x+C+\frac{\gamma}{\theta} \int_{0}^{1} \rho^{\gamma-\theta}\left|\left(\rho^{\theta}\right)_{x}\right| d x+g \\
& \leq C(T)+C(T) \int_{0}^{1} x^{\frac{2 m-2}{2 m}}\left|\left(\rho^{\theta}\right)_{x}\right| x^{-\frac{2 m-2}{2 m}} d x \\
& \leq C(T)+C(T) \int_{0}^{1} x^{2 m-2}\left[\left(\rho^{\theta}\right)_{x}\right]^{2 m} d x+C(T) \int_{0}^{1} x^{-\frac{2 m-2}{2 m-1}} d x \\
& \leq C(T),
\end{aligned}
$$

which implies (3.44).

Similarly, we have by using Young's inequality and Lemma 3.3, Corollary 3.6 that

$$
\begin{aligned}
\int_{0}^{1}\left|\rho_{x}(x, t)\right| d x & =\frac{1}{\theta} \int_{0}^{1} x^{\frac{2 m-2}{2 m}}\left|\left(\rho^{\theta}\right)_{x}\right| x^{-\frac{2 m-2}{2 m}} \rho^{1-\theta} d x \\
& \leq \frac{1}{2 m \theta} \int_{0}^{1} x^{2 m-2}\left[\left(\rho^{\theta}\right)_{x}\right]^{2 m} d x+\frac{2 m-1}{2 m \theta} \int_{0}^{1} x^{-\frac{2 m-2}{2 m-1}} \rho^{\frac{2 m(1-\theta)}{2 m-1}} d x \\
& \leq C(T)+C(T) \int_{0}^{1} x^{-\frac{2 m-2}{2 m-1}} d x \\
& \leq C(T)
\end{aligned}
$$

which implies (3.42).

This proves Lemma 3.12 .

Lemma 3.13. Under the conditions of Theorem 2.2, we have

$$
\left\{\begin{array}{l}
\int_{0}^{1}\left|u_{x}(x, t)\right| d x \leq C(T), \\
\|u(x, t)\|_{L^{\infty}([0,1] \times[0, T])} \leq C(T) .
\end{array}\right.
$$

Proof. From (3.2), we have

$$
u_{x}(x, t)=\rho^{\gamma-1-\theta}(x, t)+\rho^{-1-\theta} \int_{0}^{x} u_{t}(y, t) d y-g x \rho^{-1-\theta} .
$$


By Lemma 3.11 and Hölder's inequality, we have

$$
\begin{aligned}
\int_{0}^{1}\left|u_{x}(x, t)\right| d x & \leq \int_{0}^{1} \rho^{\gamma-1-\theta} d x+\int_{0}^{1} \rho^{-1-\theta} \int_{0}^{x}\left|u_{t}(y, t)\right| d y d x+g \int_{0}^{1} x \rho^{-1-\theta} d x \\
& \leq \int_{0}^{1} \rho^{\gamma-1-\theta} d x+\int_{0}^{1} \rho^{-1-\theta} x^{\frac{1}{2}} d x\left(\int_{0}^{1} u_{t}^{2} d x\right)^{\frac{1}{2}}+g \int_{0}^{1} x \rho^{-1-\theta} d x \\
& \leq \int_{0}^{1} \rho^{\gamma-1-\theta} d x+C(T) \int_{0}^{1} x^{\frac{1}{2}} \rho^{-1-\theta} d x
\end{aligned}
$$

The next we will prove $(3.48)_{1}$.

Case 1. If $\gamma-1-\theta<0$, then we have by Lemma 3.10

$$
\int_{0}^{1} \rho^{\gamma-1-\theta}(x, t) d x \leq C(T) \int_{0}^{1} x^{(\gamma-1-\theta)\left(1+k_{3}\right)} d x
$$

Since

$$
k_{3} \leq \frac{1}{2 \theta}-1-\frac{1}{(2 m-1) \theta}
$$

we have for $\gamma>1$

$$
(\gamma-1-\theta)\left(1+k_{3}\right) \geq-\frac{1+\theta-\gamma}{2 \theta}+\frac{1+\theta-\gamma}{(2 m-1) \theta}>-\frac{1+\theta-\gamma}{2 \theta}>-1
$$

Therefore

$$
\int_{0}^{1} \rho^{\gamma-1-\theta}(x, t) d x \leq C(T) .
$$

Case 2. If $\gamma-1-\theta \geq 0$, we can also obtain (3.51).

On the other hand, by Corollary 3.9 and Lemma 3.10, we have

$$
\begin{aligned}
\int_{0}^{1} x^{\frac{1}{2}} \rho^{-1-\theta} d x & \leq \max _{[0,1]}\left\{x^{\frac{1}{2}-\frac{1}{2 m-1}} \rho^{-\theta}\right\} \int_{0}^{1} x^{\frac{1}{2 m-1}} \rho^{-1} d x \\
& \leq C(T) \max _{[0,1]}\left\{x^{\frac{1}{2}-\frac{1}{2 m-1}} \rho^{-\theta}\right\} \\
& \leq C(T) \max _{[0,1]} x^{\frac{1}{2}-\frac{1}{2 m-1}-\theta\left(1+k_{3}\right)} .
\end{aligned}
$$

By $k_{3} \leq \frac{1}{2 \theta}-1-\frac{1}{(2 m-1) \theta}$, we have

$$
\frac{1}{2}-\frac{1}{2 m-1}-\theta\left(1+k_{3}\right) \geq 0
$$

Therefore

$$
\int_{0}^{1} x^{\frac{1}{2}} \rho^{-1-\theta} d x \leq C(T)
$$

Then (3.50), (3.51) and (3.53) show (3.48) 1 .

On the other hand, by using Sobolev's embedding theorem $W^{1,1}([0,1]) \hookrightarrow L^{\infty}([0,1])$ and Cauchy-Schwarz inequality, we have from (3.48) ${ }_{1}$ and Lemma 3.2

$$
\|u(x, t)\|_{L^{\infty}([0,1] \times[0, T])} \leq \int_{0}^{1}|u(x, t)| d x+\int_{0}^{1}\left|u_{x}(x, t)\right| d x \leq C(T) .
$$


This completes the proof of Lemma 3.13.

Our last lemma in this section is about the $L^{1}$-continuity of terms in the equations (2.1) with respect to time.

Lemma 3.14. Under the conditions of Theorem 2.2, we have for $0<s<t \leq T$ that

$$
\begin{gathered}
\int_{0}^{1}|\rho(x, t)-\rho(x, s)|^{2} d x \leq C(T)|t-s|, \\
\int_{0}^{1}|u(x, t)-u(x, s)|^{2} d x \leq C(T)|t-s|, \\
\int_{0}^{1}\left|\left(\rho^{1+\theta} u_{x}\right)(x, t)-\left(\rho^{1+\theta} u_{x}\right)(x, s)\right|^{2} d x \leq C(T)|t-s| .
\end{gathered}
$$

Proof. We first prove (3.54). To do this, using (2.1) 1 , Hölder's inequality and Lemma 3.2 , Lemma 3.3, we have

$$
\begin{aligned}
\int_{0}^{1}|\rho(x, t)-\rho(x, s)|^{2} d x & =\int_{0}^{1}\left|\int_{s}^{t} \rho_{t}(x, \eta) d \eta\right|^{2} d x \\
& =\int_{0}^{1}\left|\int_{s}^{t}\left(\rho^{2} u_{x}\right)(x, \eta) d \eta\right|^{2} d x \\
& \leq|t-s| \int_{s}^{t} \int_{0}^{1}\left(\rho^{4} u_{x}^{2}\right)(x, \eta) d x d \eta \\
& \leq|t-s| \int_{0}^{t} \max _{[0,1]}\left(\rho^{3+\theta}\right) \int_{0}^{1}\left(\rho^{1+\theta} u_{x}^{2}\right)(x, \eta) d x d \eta \\
& \leq C(T)|t-s|,
\end{aligned}
$$

which implies (3.54).

Secondly, we have by Hölder's inequality and Lemma 3.11 that

$$
\begin{aligned}
\int_{0}^{1}|u(x, t)-u(x, s)|^{2} d x & =\int_{0}^{1}\left|\int_{s}^{t} u_{t}(x, \eta) d \eta\right|^{2} d x \\
& \leq|t-s| \int_{s}^{t} \int_{0}^{1} u_{t}^{2}(x, \eta) d x d \eta \\
& \leq C(T)|t-s|
\end{aligned}
$$

So (3.55) follows.

Finally, we prove (3.56). For this, we first obtain from Hölder's inequality that

$$
\begin{aligned}
& \int_{0}^{1}\left|\left(\rho^{1+\theta} u_{x}\right)(x, t)-\left(\rho^{1+\theta} u_{x}\right)(x, s)\right|^{2} d x \\
= & \int_{0}^{1}\left|\int_{s}^{t}\left(\rho^{1+\theta} u_{x}\right)_{t}(x, \eta) d \eta\right|^{2} d x \\
\leq & |t-s| \int_{s}^{t} \int_{0}^{1}\left[\left(\rho^{1+\theta} u_{x}\right)_{t}(x, \eta)\right]^{2} d x d \eta .
\end{aligned}
$$


On the other hand, from $(2.1)_{1}$, we have

$$
\begin{aligned}
\left(\rho^{1+\theta} u_{x}\right)_{t}(x, t) & =\left(\rho^{1+\theta} u_{x t}\right)(x, t)+(1+\theta)\left(\rho^{\theta} \rho_{t} u_{x}\right)(x, t) \\
& =\left(\rho^{1+\theta} u_{x t}\right)(x, t)-(1+\theta)\left(\rho^{2+\theta} u_{x}^{2}\right)(x, t) .
\end{aligned}
$$

Therefore, using Cauchy-Schwarz inequality, we have from Lemma 3.2, Lemma 3.3, Lemma 3.11 and Lemma 3.12 that

$$
\begin{aligned}
\int_{s}^{t} \int_{0}^{1}\left[\left(\rho^{1+\theta} u_{x}\right)_{t}(x, \eta)\right]^{2} d x d \eta \leq & C \int_{0}^{t} \int_{0}^{1} \rho^{2+2 \theta} u_{x t}^{2} d x d \eta+C \int_{0}^{t} \int_{0}^{1} \rho^{4+2 \theta} u_{x}^{4} d x d \eta \\
\leq & C \int_{0}^{t} \max _{[0,1]}\left(\rho^{1+\theta}\right) \int_{0}^{1} \rho^{1+\theta} u_{x t}^{2} d x d \eta \\
& +C \int_{0}^{t} \max _{[0,1]}\left(\rho^{1+\theta} u_{x}\right)^{2} \max _{[0,1]}\left(\rho^{1-\theta}\right) \int_{0}^{1} \rho^{1+\theta} u_{x}^{2} d x d \eta \\
\leq & C(T) .
\end{aligned}
$$

This and (3.57) implies (3.56). The proof of Lemma 3.14 is completed.

\section{Construction of weak solution}

To construct a weak solution to the initial boundary value problem (2.1)-(2.4), we apply the line method as in [18], which can be described as follows. For any given positive integer $N$, let $h=\frac{1}{N}$. Discretizing the derivatives with respect to $x$ in $(2.1)$, we obtain the system of $2 N$ ordinary differential equations

$$
\left\{\begin{array}{l}
\frac{d}{d t} \rho_{2 n}^{h}(t)+\left(\rho_{2 n}^{h}(t)\right)^{2} \frac{u_{2 n+1}^{h}(t)-u_{2 n-1}^{h}(t)}{h}=0, \\
\frac{d}{d t} u_{2 n-1}^{h}(t)+\frac{P\left(\rho_{2 n}^{h}(t)\right)-P\left(\rho_{2 n-2}^{h}(t)\right)}{h} \\
=\frac{1}{h^{2}}\left\{G\left(\rho_{2 n}^{h}(t)\right)\left(u_{2 n+1}^{h}(t)-u_{2 n-1}^{h}(t)\right)-G\left(\rho_{2 n-2}^{h}(t)\right)\left(u_{2 n-1}^{h}(t)-u_{2 n-3}^{h}(t)\right)\right\}+g,
\end{array}\right.
$$

with the boundary condition

$$
\rho_{0}^{h}(t)=u_{2 N+1}^{h}(t)=0,
$$

and the initial data

$$
\left\{\begin{array}{l}
\rho_{2 n}^{h}(0)=\rho_{0}\left(2 n \cdot \frac{h}{2}\right), \\
u_{2 n-1}^{h}(0)=u_{0}\left((2 n-1) \cdot \frac{h}{2}\right),
\end{array}\right.
$$

where $n=1,2, \cdots, N$ and $G(\rho)=\mu(\rho) \rho$. When $n=1$, the second term in the right hand of $(4.1)_{2}$ is regarded as

$$
G\left(\rho_{0}^{h}(t)\right) \frac{u_{1}^{h}(t)-u_{-1}^{h}(t)}{h^{2}}=0 .
$$


To the end, we will use $\left(\rho_{2 n}, u_{2 n-1}\right)$ to replace $\left(\rho_{2 n}^{h}, u_{2 n-1}^{h}\right)$ without any ambiguity.

By using the arguments in $[19,21]$, we can prove the following lemmas for obtaining the uniform estimate of the approximate solutions to (4.1)-(4.3) with respect to $h$. Since they are the same as or similar to those in [19,21], we omit the proofs for brevity. Interested readers please refer to $[19,21]$. In the following, we consider the solutions to (4.1)-(4.3) for $0 \leq t \leq T$ where $T>0$ is any constant.

Lemma 4.1. Let $\left(\rho_{2 n}(t), u_{2 n-1}(t)\right), n=1,2, \cdots, N$, be the solution to (4.1)-(4.3). Then there exists $C(T)$ independent of $h$ such that

$$
\begin{aligned}
& \sum_{n=1}^{N}\left(\frac{1}{2} u_{2 n-1}^{2}(t)+\frac{1}{\gamma-1} \rho_{2 n}^{\gamma-1}(t)+\frac{g n h}{\rho_{2 n}(t)}\right) h+\int_{0}^{t} \sum_{n=1}^{N} G\left(\rho_{2 n}(s)\right)\left(\frac{u_{2 n+1}(s)-u_{2 n-1}(s)}{h}\right)^{2} h d s \\
& =\sum_{n=1}^{N}\left(\frac{1}{2} u_{2 n-1}^{2}(0)+\frac{1}{\gamma-1} \rho_{2 n}^{\gamma-1}(0)+\frac{g n h}{\rho_{2 n}(0)}\right) h \leq C .
\end{aligned}
$$

As a consequence of (4.4), the problem (4.1)-(4.3) has a unique global solution for any given $h$.

Lemma 4.2. For the positive integer $m$ defined by (2.5), we have

$$
\rho_{2 n}(t) \leq C(T),
$$

and

$$
\sum_{n=1}^{N} u_{2 n-1}^{2 m}(t) h+\int_{0}^{t} \sum_{n=1}^{N} u_{2 n-1}^{2 m-2}(s) \rho_{2 n}^{1+\theta}(s)\left(\frac{u_{2 n-1}(s)-u_{2 n-3}(s)}{h}\right)^{2} h d s \leq C(T) .
$$

Lemma 4.3. For the positive integer $m$ defined by (2.5), we have

$$
\begin{gathered}
\sum_{n=1}^{N}(n h)^{2 m-2}\left(\frac{\rho_{2 n}^{\theta}(t)-\rho_{2 n-2}^{\theta}(t)}{h}\right)^{2 m} h \leq C(T), \\
\sum_{n=1}^{N}(n h)^{\frac{1}{2 m-1}} \rho_{2 n}^{-1}(t) h \leq C(T), \\
\sum_{n=1}^{N}\left[\frac{d}{d t} u_{2 n-1}(t)\right]^{2} h+\int_{0}^{t} \sum_{n=1}^{N} \rho_{2 n}^{1+\theta}(s)\left(\frac{\frac{d}{d t} u_{2 n-1}(s)-\frac{d}{d t} u_{2 n-3}(s)}{h}\right)^{2} h d s \leq C(T), \\
\text { and } \rho_{2 n}(t) \geq C(T)(n h)^{1+k_{3}},
\end{gathered}
$$

where $k_{3}$ satisfies (2.11).

Based on Lemma 4.1, Lemma 4.2 and Lemma 4.3, similar to the arguments in [18, $19,21]$ and those in the proof of Lemma 3.12, Lemma 3.13, Lemma 3.14, we can get the following estimates. 
Lemma 4.4. There exists $C(T)$ such that the following estimates hold

$$
\begin{gathered}
\sum_{n=1}^{N}\left|\rho_{2 n}(t)-\rho_{2 n-2}(t)\right| \leq C(T), \\
\sum_{n=1}^{N}\left|u_{2 n+1}(t)-u_{2 n-1}(t)\right| \leq C(T), \\
\left|\rho_{2 n}^{1+\theta}(t) \frac{u_{2 n+1}(t)-u_{2 n-1}(t) \mid \leq C(T),}{h}\right| \leq u_{2 n+1}(t) \mid \leq C(T), \\
\sum_{n=1}^{N}\left|G\left(\rho_{2 n+2}(t)\right) \frac{u_{2 n+1}(t)-u_{2 n-1}(t)}{h}-G\left(\rho_{2 n}(t)\right) \frac{u_{2 n-1}(t)-u_{2 n-3}(t)}{h}\right| \leq C(T), \\
\sum_{n=1}^{N}\left|\rho_{2 n}(t)-\rho_{2 n}(s)\right|^{2} h \leq C(T)|t-s|, \\
\sum_{n=1}^{N}\left|u_{2 n-1}(t)-u_{2 n-1}(s)\right|^{2} h \leq C(T)|t-s|,
\end{gathered}
$$

and

$$
\sum_{n=1}^{N}\left|G\left(\rho_{2 n}(t)\right) \frac{u_{2 n-1}(t)-u_{2 n-3}(t)}{h}-G\left(\rho_{2 n}(s)\right) \frac{u_{2 n-1}(s)-u_{2 n-3}(s)}{h}\right|^{2} h \leq C(T)|t-s|
$$

Now we can define the sequence of approximate solutions $\left(\rho_{h}(x, t), u_{h}(x, t)\right)$ for $(x, t) \in$ $[0,1] \times[0, T]$ as follows:

$$
\begin{aligned}
& \left\{\begin{aligned}
\rho_{h}(x, t) & =\rho_{2 n}(t), \\
u_{h}(x, t) & =\frac{1}{h}\left\{\left(x-\left(n-\frac{1}{2}\right) h\right) u_{2 n+1}(t)+\left(\left(n+\frac{1}{2}\right) h-x\right) u_{2 n-1}(t)\right\},
\end{aligned}\right. \\
& \text { for }\left(n-\frac{1}{2}\right) h<x<\left(n+\frac{1}{2}\right) h . \text { Then we have for }\left(n-\frac{1}{2}\right) h<x<\left(n+\frac{1}{2}\right) h \\
& \qquad \partial_{x} u_{h}(x, t)=\frac{1}{h}\left(u_{2 n+1}(t)-u_{2 n-1}(t)\right),
\end{aligned}
$$

and

$$
\left\{\begin{array}{l}
C(T) x^{1+k_{3}} \leq \rho_{h}(x, t) \leq C(T) \\
\left|u_{h}(x, t)\right| \leq C(T), \quad \int_{0}^{1}\left|\partial_{x} u_{h}(x, t)\right| d x \leq C(T) \\
\left|G\left(\rho_{h}(x, t)\right) \partial_{x} u_{h}(x, t)\right| \leq C(T) \\
\int_{0}^{1}\left|\partial_{x}\left(G\left(\rho_{h}(x, t)\right) \partial_{x} u_{h}(x, t)\right)\right| d x \leq C(T)
\end{array}\right.
$$

By using Helly's theorem and arguments in one of the references [18, 19, 21, 28], we complete the proof of Theorem 2.2. 


\section{Uniqueness of weak solution}

In this section, we will prove the uniqueness of the weak solution constructed in Section 4. To do this, we first give two lemmas.

Lemma 5.1. For $0<\theta<\frac{1}{2}$, let

$$
l_{1}=1+\theta-\frac{1}{2\left(1+k_{3}\right)}(\leq 1)
$$

where $k_{3}$ is defined by (2.11). Then there exists some constant $C(T)$ such that

$$
\left\|\left(\rho^{l_{1}} u_{x}\right)(x, t)\right\|_{L^{\infty}([0,1] \times[0, T])} \leq C(T) .
$$

Proof. We first notice from (2.11)

$$
-\frac{1}{2}<l_{1}-1-\theta<-\theta<0 .
$$

Then from (3.2), Lemma 3.3, Lemma 3.10 and Lemma 3.11, we have by Hölder's inequality that

$$
\begin{aligned}
\left|\left(\rho^{l_{1}} u_{x}\right)(x, t)\right| & \leq \rho^{\gamma+l_{1}-1-\theta}+\rho^{l_{1}-1-\theta} \int_{0}^{x}\left|u_{t}(y, t)\right| d y+g x \rho^{l_{1}-1-\theta} \\
& \leq C(T)+\rho^{l_{1}-1-\theta}\left(\int_{0}^{x} u_{t}^{2}(y, t) d x\right)^{\frac{1}{2}} x^{\frac{1}{2}}+g x \rho^{l_{1}-1-\theta} \\
& \leq C(T)+C(T) x^{\frac{1}{2}+\left(1+k_{3}\right)\left(l_{1}-1-\theta\right)}+C x^{1+\left(1+k_{3}\right)\left(l_{1}-1-\theta\right)} .
\end{aligned}
$$

From (5.1), we have

$$
\frac{1}{2}+\left(1+k_{3}\right)\left(l_{1}-1-\theta\right)=0, \quad 1+\left(1+k_{3}\right)\left(l_{1}-1-\theta\right)=\frac{1}{2}>0 .
$$

This and (5.3) show (5.2). The proof of Lemma 5.1 is completed.

Since

$$
0 \leq \rho_{0}(x) \leq C x^{\alpha}, \quad 0<\alpha<1, \quad 0 \leq x \leq 1,
$$

therefore, similar to the proof in [30], we have the following result.

Lemma 5.2. Under conditions $(A 1)^{\prime}-(A 3)^{\prime}$, we have

$$
\rho(x, t) \leq C(T) x^{\alpha},
$$

for $0<x<1,0<t \leq T$. 
Proof of Theorem 2.4. Let $\left(\rho_{1}, u_{1}\right)(x, t)$ and $\left(\rho_{2}, u_{2}\right)(x, t)$ be two solutions to the initial boundary value problem (2.1)-(2.4) as described in Definition 2.1. Then from Lemma 5.1 and Lemma 5.2, we have for $i=1,2$

$$
\left\{\begin{array}{l}
\left\|\left(\rho_{i}^{l_{1}} \partial_{x} u_{i}\right)(x, t)\right\|_{L^{\infty}([0,1] \times[0, T])} \leq C(T), \\
0 \leq \rho_{i}(x, t) \leq C(T) x^{\alpha} .
\end{array}\right.
$$

Let

$$
\left\{\begin{array}{l}
\phi(x, t)=\left(\rho_{1}-\rho_{2}\right)(x, t) \\
\psi(x, t)=\int_{0}^{x}\left(u_{1}-u_{2}\right)(y, t) d y
\end{array}\right.
$$

for $0 \leq x \leq 1$ and $0 \leq t \leq T$.

By the boundary condition (2.2), we have

$$
\phi(0, t)=\psi(0, t)=\psi_{t}(0, t)=\psi_{x}(1, t)=0
$$

for $0 \leq t \leq T$.

In the following, we may assume that $\left(\rho_{1}, u_{1}\right)(x, t)$ and $\left(\rho_{2}, u_{2}\right)(x, t)$ are suitably smooth since the following estimates are valid for the solutions with the regularity indicated in Theorem 2.2 by using the Friedrichs mollifier.

It follows from (2.1) and (5.7)

$$
\left(\frac{\phi}{\rho_{1} \rho_{2}}\right)_{t}+\psi_{x x}=0
$$

and

$$
\psi_{t}+\frac{\rho_{1}^{\gamma}-\rho_{2}^{\gamma}}{\rho_{1}-\rho_{2}} \phi=\rho_{1}^{1+\theta} \psi_{x x}+\frac{\rho_{1}^{1+\theta}-\rho_{2}^{1+\theta}}{\rho_{1}-\rho_{2}} \phi u_{2 x} .
$$

Multiplying (5.9) by $2 \rho_{1}^{l_{2}} \rho_{2}^{-1} \phi$, we have

$$
\left(\rho_{1}^{-1+l_{2}} \rho_{2}^{-2} \phi^{2}\right)_{t}+\left(1+l_{2}\right) \rho_{1}^{l_{2}} \rho_{2}^{-2} \phi^{2} u_{1 x}+2 \rho_{1}^{l_{2}} \rho_{2}^{-1} \phi \psi_{x x}=0,
$$

where

$$
\theta \leq l_{2} \leq\left(\frac{1}{1+k_{3}}-2 \theta\right) \frac{\alpha}{1+k_{3}}-\theta
$$

The proof of the existence of $l_{2}$ is given by Remark 5.3 .

Integrating (5.11) with respect to $x$ over $[0,1]$ and using Cauchy-Schwarz inequality, we have

$$
\begin{aligned}
& \frac{d}{d t} \int_{0}^{1} \rho_{1}^{-1+l_{2}} \rho_{2}^{-2} \phi^{2} d x \\
& \quad \leq C \int_{0}^{1} \rho_{1}^{l_{2}-l_{1}} \rho_{2}^{-2} \phi^{2}\left|\rho_{1}^{l_{1}} u_{1 x}\right| d x+\frac{1}{4} \int_{0}^{1} \rho_{1}^{1+\theta} \psi_{x x}^{2} d x+C \int_{0}^{1} \rho_{1}^{2 l_{2}-1-\theta} \rho_{2}^{-2} \phi^{2} d x \\
& \quad \leq C(T) \int_{0}^{1} \rho_{1}^{l_{2}-l_{1}} \rho_{2}^{-2} \phi^{2} d x+\frac{1}{4} \int_{0}^{1} \rho_{1}^{1+\theta} \psi_{x x}^{2} d x+C \int_{0}^{1} \rho_{1}^{2 l_{2}-1-\theta} \rho_{2}^{-2} \phi^{2} d x
\end{aligned}
$$


From (5.1) and (5.12), we have

$$
l_{2}-l_{1} \geq-1+l_{2}
$$

and

$$
2 l_{2}-1-\theta \geq-1+l_{2}
$$

Therefore

$$
\frac{d}{d t} \int_{0}^{1} \rho_{1}^{-1+l_{2}} \rho_{2}^{-2} \phi^{2} d x \leq C(T) \int_{0}^{1} \rho_{1}^{-1+l_{2}} \rho_{2}^{-2} \phi^{2} d x+\frac{1}{4} \int_{0}^{1} \rho_{1}^{1+\theta} \psi_{x x}^{2} d x .
$$

Multiplying (5.10) by $\psi_{x x}$, we have

$$
\left(\frac{1}{2} \psi_{x}^{2}\right)_{t}+\rho_{1}^{1+\theta} \psi_{x x}^{2}=\frac{\rho_{1}^{\gamma}-\rho_{2}^{\gamma}}{\rho_{1}-\rho_{2}} \phi \psi_{x x}-\frac{\rho_{1}^{1+\theta}-\rho_{2}^{1+\theta}}{\rho_{1}-\rho_{2}} \phi u_{2 x} \psi_{x x}+\left(\psi_{t} \psi_{x}\right)_{x}
$$

Integrating (5.15) with respect to $x$ over $[0,1]$ and using Cauchy-Schwarz inequality, we have from (5.6), (5.8), Lemma 3.10 and noticing $l_{1}>0$

$$
\begin{aligned}
\frac{1}{2} \frac{d}{d t} & \int_{0}^{1} \psi_{x}^{2} d x+\int_{0}^{1} \rho_{1}^{1+\theta} \psi_{x x}^{2} d x \\
& =\int_{0}^{1} \frac{\rho_{1}^{\gamma}-\rho_{2}^{\gamma}}{\rho_{1}-\rho_{2}} \phi \psi_{x x} d x-\int_{0}^{1} \frac{\rho_{1}^{1+\theta}-\rho_{2}^{1+\theta}}{\rho_{1}-\rho_{2}} \phi u_{2 x} \psi_{x x} d x \\
& \leq \frac{1}{4} \int_{0}^{1} \rho_{1}^{1+\theta} \psi_{x x}^{2} d x+C(T) \int_{0}^{1} \rho_{1}^{-1-\theta} \phi^{2} d x+C(T) \int_{0}^{1} \rho_{1}^{-1-\theta} \phi^{2} u_{2 x}^{2} d x \\
& \leq \frac{1}{4} \int_{0}^{1} \rho_{1}^{1+\theta} \psi_{x x}^{2} d x+C(T) \max _{[0,1]}\left(\rho_{1}^{-\theta-l_{2}} \rho_{2}^{2}\right) \int_{0}^{1} \rho_{1}^{-1+l_{2}} \rho_{2}^{-2} \phi^{2} d x \\
& +C(T) \max _{[0,1]}\left(\rho_{1}^{-\theta-l_{2}} \rho_{2}^{2} u_{2 x}^{2}\right) \int_{0}^{1} \rho_{1}^{-1+l_{2}} \rho_{2}^{-2} \phi^{2} d x \\
& \leq \frac{1}{4} \int_{0}^{1} \rho_{1}^{1+\theta} \psi_{x x}^{2} d x+C(T) \max _{[0,1]}\left(\rho_{1}^{-\theta-l_{2}} \rho_{2}^{2}\right) \int_{0}^{1} \rho_{1}^{-1+l_{2}} \rho_{2}^{-2} \phi^{2} d x \\
& +C(T) \max _{[0,1]}\left(\rho_{1}^{-\theta-l_{2}} \rho_{2}^{2-2 l_{1}}\right) \max _{[0,1]}\left(\rho_{2}^{l_{1}} u_{2 x}\right)^{2} \int_{0}^{1} \rho_{1}^{-1+l_{2}} \rho_{2}^{-2} \phi^{2} d x \\
& \leq \frac{1}{4} \int_{0}^{1} \rho_{1}^{1+\theta} \psi_{x x}^{2} d x+C(T) \max _{[0,1]}\left(x^{\left(2-2 l_{1}\right) \alpha-\left(\theta+l_{2}\right)\left(1+k_{3}\right)}\right) \int_{0}^{1} \rho_{1}^{-1+l_{2}} \rho_{2}^{-2} \phi^{2} d x .
\end{aligned}
$$

From (5.1) and (5.12), we have

$$
\left(2-2 l_{1}\right) \alpha-\left(\theta+l_{2}\right)\left(1+k_{3}\right) \geq 0 .
$$

Therefore

$$
\begin{aligned}
& \frac{1}{2} \frac{d}{d t} \int_{0}^{1} \psi_{x}^{2} d x+\int_{0}^{1} \rho_{1}^{1+\theta} \psi_{x x}^{2} d x \\
& \quad \leq \frac{1}{4} \int_{0}^{1} \rho_{1}^{1+\theta} \psi_{x x}^{2} d x+C(T) \int_{0}^{1} \rho_{1}^{-1+l_{2}} \rho_{2}^{-2} \phi^{2} d x
\end{aligned}
$$


Thus (5.14) and (5.17) show

$$
\begin{aligned}
& \frac{d}{d t}\left(\int_{0}^{1} \rho_{1}^{-1+l_{2}} \rho_{2}^{-2} \phi^{2} d x+\frac{1}{2} \int_{0}^{1} \psi_{x}^{2} d x\right)+\frac{1}{2} \int_{0}^{1} \rho_{1}^{1+\theta} \psi_{x x}^{2} d x \\
& \quad \leq C(T) \int_{0}^{1} \rho_{1}^{-1+l_{2}} \rho_{2}^{-2} \phi^{2} d x .
\end{aligned}
$$

By using Gronwall's inequality, we have for any $t>0$

$$
\int_{0}^{1} \rho_{1}^{-1+l_{2}} \rho_{2}^{-2} \phi^{2} d x=0
$$

and

$$
\int_{0}^{1} \psi_{x}^{2} d x=0
$$

This proves Theorem 2.4.

Remark 5.3. $\quad$ There exists some constant $l_{2}$ which satisfies (5.12).

In fact, (5.12) is equivalent to

$$
\left(1+k_{3}\right)^{2}+\left(1+k_{3}\right) \alpha-\frac{\alpha}{2 \theta} \leq 0
$$

which implies

$$
1+k_{3} \leq-\frac{\alpha}{2}+\frac{1}{2} \sqrt{\alpha^{2}+\frac{2 \alpha}{\theta}} .
$$

From (2.11) and (5.22), we have

$$
\frac{2 m-1}{2 m-2 m \theta-1}+\frac{\alpha}{2} \leq \frac{1}{2} \sqrt{\alpha^{2}+\frac{2 \alpha}{\theta}}
$$

i.e.,

$$
\alpha \geq \frac{2(2 m-1)^{2} \theta}{(2 m-2 m \theta-1)(2 m-6 m \theta+2 \theta-1)} .
$$

Furthermore, (5.24) can be rewritten as

$$
4\left(3 \alpha \theta^{2}-(4 \alpha+2) \theta+\alpha\right) m^{2}-4\left(\alpha \theta^{2}-(3 \alpha+2) \theta+\alpha\right) m+\alpha-(2 \alpha+2) \theta \geq 0 .
$$

When $0<\theta<\frac{\alpha}{1+2 \alpha+\sqrt{1+4 \alpha+\alpha^{2}}}$, we have

$$
3 \alpha \theta^{2}-(4 \alpha+2) \theta+\alpha>0 .
$$

From the assumption $(\mathrm{A} 3)^{\prime}$ and (5.26), we see (5.25) holds. Now let

$$
\frac{2 m \theta}{2 m(1-\theta)-1} \leq k_{3} \leq \min \left\{\frac{1}{2 \theta}-1-\frac{1}{(2 m-1) \theta},-\frac{\alpha}{2}-1+\frac{1}{2} \sqrt{\alpha^{2}+\frac{2 \alpha}{\theta}}\right\}
$$

that is to say, we may choose $m$ so large that (2.14) is satisfied. Then (5.22) holds. So there exists some constant $l_{2}$ such that (5.12) hold. 
Remark 5.4. When $\alpha$ is sufficiently close to 1 , then $\theta$ may be sufficiently close to $1-\frac{\sqrt{6}}{3}$.

Acknowledgement: The research of the second author was supported by Hong Kong RGC Competitive Earmarked Research Grant CityU 102703 and the National Natural Science Foundation of China \#10329101, respectively. The research of the third author was supported by Program for New Century Excellent Talents in University \#NCET-040745, the Key Project of the National Natural Science Foundation of China \#10431060 and the Key Project of Chinese Ministry of Education \#104128.

\section{References}

[1] R. Balian, From microphysics to macrophysics, Texts and monographs in physics, Springer, 1982.

[2] G.Q. Chen, D. Hoff and K. Trivisa, Global solutions of the compressible Navier-Stokes equations with large discontinuous initial data, Comm. Partial Differential Equations, $25(2000), 2233-2257$.

[3] G.Q. Chen and M. Kratka, Global solutions to the Navier-Stokes equations for compressible heat-conducting flow with symmetry and free boundary, Comm. Partial Differential Equations, 27(2002), 907-943.

[4] D.Y. Fang, T. Zhang, A note on compressible Navier-Stokes equations with vacuum state in one dimension, Nonlinear Anal., TMA, 58(2004), 719-731.

[5] H. Grad, Asymptotic theory of the Boltzmann equation II. In: Rarefied gas dynamics, 1(ed. J. Laurmann), New York Academic Press(1963), 26-59.

[6] D. Hoff, Strong convergence to global solutions for multidimensional flows of compressible, viscous fluids with polytropic equations of state and discontinuous initial data, Arch. Rat. Mech. Anal., 132(1995), 1-14.

[7] D. Hoff and T.-P. Liu, The inviscid limit for the Navier-Stokes equations of compressible isentropic flow with shock data, Indiana Univ. Math. J., 38(1989), 861-915.

[8] D. Hoff and D. Serre, The failure of continuous dependence on initial data for the Navier-Stokes equations of compressible flow, SIAM J. Appl. Math., 51(1991), 887898.

[9] D. Hoff and J. Smoller, Non-formation of vacuum states for compressible NavierStokes equations, Comm. Math. Phys., 216(2001), 255-276.

[10] S. Jiang, Global smooth solutions of the equations of a viscous, heat-conducting, onedimensional gas with density-dependent viscosity, Math. Nachr., 190(1998), 169-183. 
[11] S. Jiang, Z.P. Xin and P. Zhang, Global weak solutions to $1 D$ compressible isentropic Navier-Stokes equations with density-dependent viscosity, preprint 1999.

[12] S. Kawashima and T. Nishida, The initial-value problems for the equations of viscous compressible and perfect compressible fluids, RIMS, Kokyuroku 428, Kyoto University, Nonlinear Functional Analysis, June 1981, 34-59.

[13] P.L. Lions, Mathematical Topics in Fluid Mechanics, Vol. 1, 2, (1998), Clarendon Press-Oxford.

[14] T.-P. Liu, Z. Xin and T. Yang, Vacuum states of compressible flow, Discrete and Continuous Dynamical Systems, 4(1998), 1-32.

[15] T. Luo, Z. Xin and T. Yang, Interface behavior of compressible Navier-Stokes equations with vacuum, SIAM J. Math. Anal., 31(2000), 1175-1191.

[16] T. Makino, On a local existence theorem for the evolution equations of gaseous stars, "Patterns and wave-qualitative analysis of nonlinear differential equations", Ed. T. Nishida, M. Mimura and H. Fujii, North-Holland, 1986, 459-479.

[17] T. Nishida, Equations of fluid dynamics-free surface problems, Comm. Pure Appl. Math., XXXIX(1986), 221-238.

[18] T. Nishida, Equations of motion of compressible viscous fluids, Patterns and WavesQualitative Analysis of Nonlinear Differential Equations, (1986), 97-128.

[19] M. Okada, Free boundary value problems for the equation of one-dimensional motion of viscous gas, Japan J. Appl. Math., 6(1989), 161-177.

[20] M. Okada and T. Makino, Free boundary problem for the equations of spherically symmetrical motion of viscous gas, Japan J. Indust. Appl. Math., 10(1993), 219-235.

[21] M. Okada, Š. Matušù-Nečasová and T. Makino, Free boundary problem for the equation of one-dimensional motion of compressible gas with density-dependent viscosity, Ann. Univ. Ferrara Sez, VII-Sc.Mat., 48(2002), 1-20.

[22] D. Serre, Sur l'equation mondimensionnelle d'un fluide visqueux, compressible et conducteur de chaleur, Comptes rendus Acad. des Sciences. 303(1986), 703-706.

[23] I. Straškraba, A.A. Zlotnik, Global properties of solutions to 1D-viscous compressible baratropic fluid equations with density dependent viscosity, Z. angew. Math. Phys., 54(2003), 593-607.

[24] I. Straškraba, Global analysis of 1-D Navier-Stokes equations with density dependent viscosity, In: Navier-Stokes equations and related nonlinear problems (H. Amann et al., eds.) VSP Utrecht, TEV Vilnius (1997), 371-390. 
[25] Z. Xin, Blow-up of smooth solutions to the compressible Navier-Stokes equations with compact density, Comm. Pure Appl. Math., 51(1998), 229-240.

[26] S.W. Vong, T. Yang and C.J. Zhu, Compressible Navier-Stokes equations with degenerate viscosity coefficient and vacuum (II), J. Differential Equations, 192(2003), 475-501.

[27] T. Yang, Some recent results on compressible flow with vacuum, Taiwanese J. Math., $4(2000), 33-44$.

[28] T. Yang, Z.A. Yao and C.J. Zhu, Compressible Navier-Stokes equations with densitydependent viscosity and vacuum, Comm. Partial Differential Equations, 26(2001), 965-981.

[29] T. Yang and H.J. Zhao, A vacuum problem for the one-dimensional compressible Navier-Stokes equations with density-dependent viscosity, J. Differential Equations, 184(2002), 163-184.

[30] T. Yang and C.J. Zhu, Compressible Navier-Stokes equations with degenerate viscosity coefficient and vacuum, Comm. Math. Phys., 230(2002), 329-363.

[31] A.A. Zlonik, Uniform estimates and stabilization of symmetric solutions of a system of quasilinear equations, Diff. Equations 36(2000), 701-716. 